Pacific Journal of Mathematic 


\title{
QUASI-SIMILARITY FOR SPECTRAL OPERATORS ON BANACH SPACES
}

\author{
L. TZAFRIRI
}

The unitary equivalence for normal operators is generalized for a large class of spectral operators on Banach spaces. This generalization which is called quasi-similarity coincides with the semi-similarity introduced by Feldzamen in the particular case of Hilbert spaces and for it the Weyr characteristic forms a complete set of invariants.

The spectral representation theorem for normal operators asserts that every Hilbert space admits a spectral representation relative to an arbitrary bounded normal operator defined in it. This means that every normal operator on a Hilbert space can be represented, through a unitary transformation (similarity), as the "multiplication" operator on an appropriate direct sum of $L_{2}$-spaces (e.g. see [4] X-5). An immediate consequence of this result consists in the possibility of dividing the normal operators into classes of unitarily equivalent operators.

The case of spectral operators on Hilbert spaces is more complicated and it was studied by Feldzamen in his laborious paper [5]. He has defined the Weyr and Segre characteristics for spectral operators of finite multiplicity and shown that they are similarity invariants but not a complete set of invariants. By introducing a generalization of the similarity called semi-similarity (roughly, it is a decomposition of the identity, by means of projections in the respective resolutions of the identity, into similar parts) he has obtained an equivalence relation for spectral operators of finite multiplicity on Hilbert spaces for which the Weyr (or Segre) characteristic provides a complete set of invariants.

The purpose of this paper is to construct a quasi-similarity theory for spectral operators on Banach spaces which coincides with the usual similarity for normal operators or with Feldzamen's semi-similarity in the case of the spectral operators of finite multiplicity on Hilbert spaces. One of the basic properties of quasi-similarity is the fact that for it as well as for semi-similarity, the Weyr characteristic is a complete set of invariants. The multiplicity of the projections in the resolution of the identity (defined by Bade in [2]) is also an invariant of quasi-similarity.

A beginning in this direction was done by Bade [2] Theorem 9.2 who has shown that a spectral operator of scalar type on a separable Banach space whose resolution of the identity contains no projections of infinite uniform multiplicity, can be represented, through a densely 
defined closed linear map with densely defined inverse, as the "multiplication" operator on a suitable direct sum of $L_{1}$-spaces. It should be mentioned that our results will be proved under the same restrictions concerning the underlying space and the multiplicity of the projections.

2. Quasi-similarity. The similarity equivalence in the sense of the afore-mentioned theorem of Bade consists in essence in the existence of a densely defined closed linear map having a densely defined inverse (instead of a unitary transformation in the Hilbert case). Since such a similarity seems to be insufficient in order to insure the existence of a large set of natural invariants we should add some supplementary conditions which, of course, are satisfied by the map defined in Bade's theorem.

In this section $A_{1}$ and $A_{2}$ will be two bounded linear spectral operators of finite type on the separable Banach spaces $X_{1}$ and $X_{2}$ whose resolutions of the identity are $E_{1}(\cdot)$ and $E_{2}(\cdot)$ respectively.

Since in most cases we are dealing in fact only with spectral operators for which there are no projections of infinite uniform multiplicity in their resolutions of the identity, it follows from [10] Theorem 4 that separability is only a slight restriction equivalent with the countable chain condition (for details concerning the multiplicity theory for Boolean algebras of projections on Banach spaces see [2]).

Definition 1. We shall say that $A_{2}$ is quasi-similar to $A_{1}$ if there exists a densely defined closed linear map $\tau: X_{1} \rightarrow X_{2}$ with densely defined inverse $\left(D(\tau)\right.$ and $D\left(\tau^{-1}\right)$ will denote the respective domains of $\tau$ and $\tau^{-1}$ ) such that:

(i) $\tau A_{1} \tau^{-1} y=A_{2} y ; y \in D\left(\tau^{-1}\right)$

(ii) For every Borel set $\delta$ on the complex plane there is a constant $M(\delta)$ such that

$$
\left\|\tau E_{1}(\delta) \tau^{-1} y\right\| \leqq M(\delta)\|y\| ; y \in D\left(\tau^{-1}\right) .
$$

Let us remark that in view of (i) and (ii), $A_{1} D(\tau) \subseteq D(\tau)$ and also $E_{1}(\delta) D(\tau) \subseteq D(\tau)$ for every Borel set $\delta$.

Lemma 2. If $A_{2}$ is quasi-similar to $A_{1}$, then there is a constant $M$ such that

$$
\left\|\tau \int f(\lambda) E_{1}(d \lambda) \tau^{-1} y\right\| \leqq M\|y\| E_{1}-\operatorname{ess} \sup _{\lambda}|f(\lambda)|
$$

for every bounded Borel function $f$ and $y \in D\left(\tau^{-1}\right)$. 
Proof. Let us denote

$$
F(\delta) y=\tau E_{1}(\delta) \tau^{-1} y ; y \in D\left(\tau^{-1}\right), \delta \in \text { Borel sets. }
$$

Then $F(\delta)$ is a bounded idempotent operator densely defined on $X_{2}$. Thus $F(\delta)$ can be extended in a unique way on the whole space $X_{2}$ such that it will be a bounded projection. Evidently, $F(\cdot)$ forms a Boolean algebra of projections and $E_{1}(\delta) \leqq E_{1}(\delta)$ if and only if $F(\delta) \leqq$ $F(\sigma)$ for every pair of Borel sets $\delta$ and $\sigma$. Consequently, $F(\cdot)$ is a Boolean algebra of projections, $\sigma$-complete as an abstract Boolean algebra and by [1] Theorem 2.2 it is bounded, i.e. there exists a constant $M_{0}$ such that

$$
\|F(\delta) y\|=\left\|\tau E(\delta) \tau^{-1} y\right\| \leqq M_{0}\|y\| ; y \in D\left(\tau^{-1}\right) ; \delta \in \text { Borel sets. }
$$

Now, let $f$ be a bounded Borel function and $\left\{f_{n}\right\}$ a sequence of finitely valued functions converging uniformly to $f$ on $\sigma\left(A_{1}\right)$ (the spectrum of $A_{1}$ is the support of $E_{1}(\cdot)$ and $\left.F(\cdot)\right)$. Then

$$
\lim _{n \rightarrow \infty} \int_{\sigma\left(A_{1}\right)} f_{n}(\lambda) E_{1}(d \lambda) \tau^{-1} y=\int_{\sigma\left(A_{1}\right)} f(\lambda) E_{1}(d \lambda) \tau^{-1} y ; y \in D\left(\tau^{-1}\right)
$$

and

$$
\lim _{n \rightarrow \infty} \tau \int_{\sigma\left(A_{1}\right)} f_{n}(\lambda) E_{1}(d \lambda) \tau^{-1} y=\int_{\sigma\left(A_{1}\right)} f(\lambda) F(d \lambda) y ; \quad y \in D\left(\tau^{-1}\right) .
$$

Since $\tau$ is closed it follows that

$$
\int f(\lambda) E_{1}(d \lambda) D(\tau) \leqq D(\tau)
$$

for every bounded Borel function $f$ and by [3] p. 341

$$
\begin{aligned}
\left\|\tau \int f(\lambda) E_{1}(d \lambda) \tau^{-1} y\right\| & =\left\|\int f(\lambda) F(d \lambda) y\right\| \\
& \leqq 4 M_{0}\|y\| E_{1}-\left.\operatorname{ess} \sup \right|_{\lambda} f(\lambda) \mid ; y \in D\left(\tau^{-1}\right)
\end{aligned}
$$

(we have used the fact that $E_{1}(\delta)=0$ if and only if $F(\delta)=0$ for some Borel set $\delta$ ).

Theorem 3. Let $A_{1}=S_{1}+N_{1}, A_{2}=S_{2}+N_{2}$ be the respective canonical decompositions of $A_{1}$ and $A_{2}$ (see [3] p. 334). If $A_{2}$ is quasi-similar to $A_{1}$ then:

(a) $\tau E_{1}(\delta) \tau^{-1} y=E_{2}(\delta) y ; y \in D\left(\tau^{-1}\right) ; \delta \in$ Borel sets.

(b) $A_{1}$ is quasi-similar to $A_{2}$, i.e. quasi-similarity is a symmetric relation.

(c) $S_{1}$ and $S_{2}$ are quasi-similar.

(d) $N_{1}$ and $N_{2}$ are quasi-similar nilpotents of the same order. 
(e) $f\left(S_{1}\right)$ and $f\left(S_{2}\right)$ are quasi-similar for every bounded Borel function $f$.

Proof. We have observed in the proof of the previous lemma that the family of projections $F(\cdot)$ defined by (2.3) forms a bounded Boolean algebra of projections. Moreover, it is $\sigma$-complete as an abstract Boolean algebra of projections. Obviously, it commutes with $A_{2}$ and therefore with $E_{2}(\cdot)$.

Now, let us denote

$$
S_{3} y=\tau S_{1} \tau^{-1} y ; N_{3} y=\tau N_{1} \tau^{-1} y ; y \in D\left(\tau^{-1}\right) .
$$

The definition of $S_{3}$ and $N_{3}$ is possible in view of (2.7) and it is easy to see that $N_{3}$ is a nilpotent of the same order as $N_{1}$ and

$$
S_{3}=\int \lambda F(d \lambda)
$$

Hence

$$
A_{2}=S_{2}+N_{2}=S_{3}+N_{3}
$$

and all these operators commute. Thus $S_{3}$ is a spectral operator and $\sigma\left(S_{3}\right)=\sigma\left(S_{2}\right)=\sigma\left(A_{2}\right)$. Consequently,

$$
\sigma\left(A_{2} / F(\delta) X_{2}\right)=\sigma\left(S_{3} / F(\delta) X_{2}\right) \leqq \bar{\delta} ; \delta \in \text { Borel sets },
$$

i.e. $F(\cdot)$ is a nonnecessarily countably additive "resolution of the identity" for the spectral operator $A_{2}$.

By [3] Theorem 4, for every closed set $\delta$

$$
E_{2}(\delta) X_{2}=\left\{y \mid y \in X_{2}, \sigma_{A_{2}}(y) \leqq \delta\right\}
$$

where $\sigma_{A_{2}}(y)$ denotes the spectrum of $y$ with respect to $A_{2}$ (see [3] p. 327). One can easily see that in order to prove the inclusion $E_{2}(\delta) X_{2} \subseteq\left\{y \mid y \in X_{2}, \sigma_{A_{2}}(y) \subseteq \delta\right\}$, Dunford does not use the countable additivity of the resolution of the identity. Thus

$$
F(\delta) X_{2} \leqq\left\{y \mid y \in X_{2}, \sigma_{A_{2}}(y) \leqq \delta\right\}
$$

and further $F(\delta) X_{2} \subseteq E_{2}(\delta) X_{2}$ which implies

$$
F(\delta) \leqq E_{2}(\delta)
$$

for every closed set $\delta$.

Let $\sigma$ be a Borel set, $\sigma^{\prime}$ its complement and $\partial \sigma$ its boundary. Suppose that $E_{2}(\partial \sigma) y=0$ for some Borel set $\sigma$ and $y \in X_{2}$. Then, by (2.10) we have $F(\partial \sigma) y=0$ since $\partial \sigma$ is a closed set. Hence,

$$
y=E_{2}(\bar{\sigma}) y+E_{2}\left(\bar{\sigma}^{\prime}\right) y=F(\bar{\sigma}) y+F\left(\bar{\sigma}^{\prime}\right) y
$$


and by $(2.10)$

$$
E_{2}(\bar{\sigma}) y=E_{2}(\bar{\sigma}) F(\bar{\sigma}) y+E_{2}(\bar{\sigma}) F\left(\bar{\sigma}^{\prime}\right) y=F(\bar{\sigma}) y .
$$

In conclusion we get

$$
E_{2}(\sigma) y=F(\sigma) y \text {. }
$$

Let $\delta$ be a closed set and define

$$
\sigma_{\nu}=\{\mu|| \mu-\lambda \mid<\nu \text { for some } \lambda \in \delta\} ; 0<\nu .
$$

In view of the separability of $X_{2}$ one can find a sequence $\left\{y_{n}\right\}$ dense in $X_{2}$. By [1] Theorem 3.1, to every $y_{n}$ there is a linear functional $y_{n}^{*} \in X_{2}$ for which $y_{n}^{*} E_{2}(\sigma) y_{n} \geqq 0 ; \sigma \in$ Borel sets and $y_{n}^{*} E_{2}(\sigma) y_{n}=0$ for some $\sigma$ implies $E_{2}(\sigma) y_{n}=0$. Let us put

$$
g_{n}(\nu)=y_{n}^{*} E_{2}\left(\sigma_{\nu}\right) y_{n} ; 0<\nu ; \quad n=1,2, \cdots .
$$

Evidently, $\mathrm{g}_{n}(\nu)$ is an increasing function of $\nu$ whose set $D_{n}$ of discontinuities is at most countable. Therefore, the set $D=\bigcup_{n=1}^{\infty} D_{n}$ is also at most countable. If $\nu_{0} \in D$, then

$$
\lim _{\nu \rightarrow \nu_{0}} y_{n}^{*} E_{2}\left(\sigma_{\nu}\right) y_{n}=y_{n}^{*} E_{2}\left(\sigma_{\nu_{0}}\right) y_{n} ; \quad n=1,2, \cdots .
$$

and further $y_{n}^{*} E_{2}\left(\bar{\sigma}_{\nu_{0}}\right) y_{n}=y_{n}^{*} E_{2}\left(\sigma_{\nu_{0}}\right) y_{n}$. Thus, it follows

$$
E_{2}\left(\bar{\sigma}_{\nu_{0}}\right)=E_{2}\left(\sigma_{\nu_{0}}\right)
$$

which implies $E_{2}\left(\partial \sigma_{\nu}\right)=0 ; \nu \in D$. Let us consider a decreasing sequence $\left\{\nu_{k}\right\}$ such that $\nu_{k} \rightarrow 0$ when $k \rightarrow \infty$ and $\nu_{k} \notin D$. By (2.11) we have

$$
E_{2}\left(\sigma_{\nu_{k}}\right)=F\left(\sigma_{\nu_{k}}\right) \text {; }
$$$$
k=1,2, \cdots \text {. }
$$

Observe that $\bigcap_{k=1}^{\infty} \sigma_{\nu_{k}}=\delta$ which implies

$$
\bigwedge_{k=1}^{\infty} E_{1}\left(\sigma_{\nu_{k}}\right)=E_{1}(\delta) ; \bigwedge_{k=1}^{\infty} E_{2}\left(\sigma_{\nu_{k}}\right)=E_{2}(\delta) \text {. }
$$

Now, using the fact that $E_{1}(\cdot)$ and $E_{2}(\cdot)$ are strongly countable additive spectral measures and (2.12) we shall have

$$
\begin{aligned}
E_{1}(\delta) \tau^{-1} y & =\lim _{k \rightarrow \infty} E_{1}\left(\sigma_{\nu_{k}}\right) \tau^{-1} y & & y \in D\left(\tau^{-1}\right) \\
E_{2}(\delta) y & =\lim _{k \rightarrow \infty} E_{2}\left(\sigma_{\nu_{k}}\right) y=\lim _{k \rightarrow \infty} F\left(\sigma_{\nu_{k}}\right) y=\lim _{k \rightarrow \infty} \tau E_{1}\left(\sigma_{\nu_{k}}\right) \tau^{-1} y ; & & y \in D\left(\tau^{-1}\right) .
\end{aligned}
$$

Then, since $\tau$ is closed, we can conclude

$$
E_{2}(\delta) y=\tau E_{1}(\delta) \tau^{-1} y=F(\delta) y ;
$$

and further 


$$
E_{2}(\delta)=F(\delta)
$$

for every closed set $\delta$ in the complex plane and, consequently, for every open set $\delta$.

Finally, we shall use the regularity of the spectral measure $E_{2}(\cdot)$. Let $\eta$ be a Borel set; for every $y \in X_{2}$ there is a sequence of closed sets $\left\{\delta_{n}\right\}$ and a sequence of open sets $\left\{\sigma_{n}\right\}$ such that $\delta_{n} \subseteq \eta \sqsubseteq \sigma_{n} ; n=$ $1,2, \cdots$ and

$$
\lim _{n \rightarrow \infty} E_{2}\left(\sigma_{n}-\delta_{n}\right) y=0
$$

By (2.13)

$$
\lim _{n \rightarrow \infty} F\left(\sigma_{n}-\delta_{n}\right) y=0
$$

and therefore $F(\eta)=E_{2}(\eta)$ which proves the first part of the theorem. The part (b) follows from the fact that

$$
\left\|\tau^{-1} E_{2}(\delta) \tau x\right\|=\left\|E_{1}(\delta) x\right\| \leqq K\|x\| ;
$$

where $K$ is a bound for the resolution of the identity of $A_{1}$. Furthermore, the part (e) follows from (2.5), (2.6) and part (a) of this theorem. Then $(c)$ and $(d)$ are trivial consequences.

COROLLARY 4. The spectrum and its fine structure are quasisimilarity invariants (for spectral operators of finite type).

Proof. By the previous theorem $E_{1}(\cdot)$ and $E_{2}(\cdot)$ have the same support, therefore $\sigma\left(A_{1}\right)=\sigma\left(A_{2}\right)$. Let us point out that Foguel [6] Theorem 1, p. 56, has proved that a spectral operator of finite type has no residual spectrum and a point $\lambda$ belongs to its point spectrum if and only if $E(\{\lambda\}) \neq 0$. Then, the last statement follows from the fact that in our case $E_{1}(\{\lambda\}) \neq 0$ whenever $E_{2}(\{\lambda\}) \neq 0$.

The concept of multiplicity as used in what follows was introduced by Bade in [2]. For convenience we shall summarize here some notations and results concerning. the theory of multiplicity for Boolean algebras of projections on Banach spaces.

Let $\mathfrak{B}$ be a Boolean algebra of projections and remark that it may be considered as a spectral measure $E(\cdot)$ on its own Stone space $\Omega$. The cyclic subspace $\mathfrak{M}(x)$ generated by a vector $x$ is $\operatorname{clm}\{E x \mid E \in \mathfrak{B}\}$. For each $x$ the projection $C(x)=\wedge\{E \mid E x=x\}$ will be called the carrier projection of $x$. If the underlying space is separable then every complete Boolean algebra of projections is countably decomposable, i.e., every family of disjoint projections in $\mathfrak{B}$ is at most countable. In this case the multiplicity of $E \in \mathfrak{B}$ will be defined as the smallest cardinal power of a set $A$ of vectors such that $E X=\operatorname{clm}\{\mathfrak{M}(x) \mid x \in A\}$. 
We say $E \in \mathfrak{B}$ has uniform multiplicity $n$ if the multiplicity of $F \in \mathfrak{B}$ is equal to $n$ whenever $0 \neq F \leqq E$.

In order to prove the invariance of the multiplicity under quasisimilarity we should use the notion of independence of vectors with respect to a Boolean algebra of projections which was introduced by Feldzamen in [5]. A finite family of nonzero vectors $\left\{x_{1}, \cdots, x_{m}\right\}$ will be called independent if there is a family of vectors $\left\{z_{1}, \cdots, z_{m}\right\}$ such that

(a) $\left.\mathfrak{M}\left(x_{\imath}\right) \subseteq \mathfrak{M}\left(z_{i}\right)\right\}$

(b) $C\left(z_{i}\right)=I \quad i=1,2, \cdots, m$.

(c) $\sum_{i=1}^{m} \int f_{i}(\omega) E(d \omega) z_{i}=0$ implies $\int f_{i}(\omega) E(d \omega)=0$ for $i=1,2, \cdots, m$.

Finally, let us remark that in our case when the underlying space is separable the multiplicity of a projection cannot be greater than $\aleph_{*}$.

THEOREM 5. The multiplicity is a quasi-similarity invariant i.e. the multiplicity of $E_{1}(\delta)$ is equal to the multiplicity of $E_{2}(\delta)$ for every Borel set $\delta$ in the complex plane.

Proof. First, assume that the identity in $X_{1}$ has finite uniform multiplicity $n$ and consider all the finite families of independent (with respect to the Boolean algebra $\left.E_{2}(\cdot)\right)$ vectors $\left\{y_{1}, \cdots, y_{p}\right\}$;

$$
y_{i} \in D\left(\tau^{-1}\right) \subseteq X_{2}, \bigwedge_{i=1}^{p} C\left(y_{\imath}\right) \neq 0 ; \quad i=1, \cdots, p .
$$

For such a family denote $x_{i}=\tau^{-1} y_{i} ; i=1, \cdots, p$. Obviously, $C\left(x_{i}\right) x=\tau^{-1} C\left(y_{i}\right) \tau x ; x \in D(\tau)$ which implies $\bigwedge_{i=1}^{p} C\left(x_{i}\right) \neq 0$. Assume that

$$
\sum_{\imath=1}^{p} \int f_{\imath}(\lambda) E_{1}(d \lambda) x_{\imath}=0
$$

for some bounded Borel functions $f_{i} ; i=1, \cdots, n$. Then, by applying $\tau$ and using Theorem 3 part (e) we get

$$
\sum_{\imath=1}^{p} \int f_{i}(\lambda) E_{2}(d \lambda) y_{\imath}=0
$$

and by $\lfloor 5\rfloor$ Lemma 2

$$
\int f_{2}(\lambda) E_{2}(d \lambda) y_{i}=0 ; \quad i=1, \cdots, p .
$$

Thus, by Theorem 3 part (e) we have

$$
\int f_{i}(\lambda) E_{1}(d \lambda) x_{i}=0 ; \quad i=1, \cdots, p
$$


and then, by [5] Theorem 8 , the family $\left\{x_{1}, \cdots, x_{p}\right\}$ will be independent (with respect to the Boolean algebra of projections $E_{1}(\cdot)$ ). In view of [5] Theorem 6 this is possible only if $p \leqq n$.

Let $\left\{y_{1}, \cdots, y_{p}\right\}$ be a family of independent vectors; $y_{i} \in D\left(\tau^{-1}\right)$, $E_{2}\left(\delta_{0}\right)=\bigwedge_{i=1}^{p} C\left(y_{i}\right) \neq 0 ; i=1, \cdots, p$ for which $p$ is maximal. By [2] Lemma 4.1, for every $y \in D\left(\tau^{-1}\right)$ there exists a maximal projection $E_{2}(\sigma)$ such that

$$
E_{2}(\sigma) E_{2}\left(\delta_{0}\right) y \in \bigvee_{i=1}^{p} \mathfrak{M}\left(E_{2}\left(\delta_{0}\right) y_{i}\right)
$$

and

$$
\bigvee_{i=1}^{p} \mathfrak{M} \mathcal{L}\left(E_{2}\left(\delta_{0}\right) y_{i}\right) \cap \mathfrak{M} \mathcal{L}\left(\left(I-E_{2}(\sigma)\right) E_{2}\left(\delta_{0}\right) y\right)=\{0\}
$$

In view of the definition of quasi-similarity and Theorem 3

$$
y_{0}=\left(I-E_{2}(\sigma)\right) E_{2}\left(\delta_{0}\right) y \in D\left(\tau^{-1}\right)
$$

and if $y_{0} \neq 0$ then $\Lambda_{i=0}^{p} C\left(y_{i}\right)=C\left(y_{0}\right) \neq 0$ and by (2.14) $\left\{y_{0}, y_{1}, \cdots, y_{p}\right\}$ will be an independent family of vectors from $D\left(\tau^{-1}\right)$ which contradicts the maximality of $p$. Hence $y_{0}=0$ and consequently

$$
E_{2}\left(\delta_{0}\right) y=E_{2}(\sigma) E_{2}\left(\delta_{0}\right) y \in \bigvee_{i=1}^{p} \mathfrak{M}\left(E_{2}\left(\delta_{0}\right) y_{i}\right)
$$

for every $y \in D\left(\tau^{-1}\right)$. Since $D\left(\tau^{-1}\right)$ is dense it will follow that the multiplicity of $E_{2}\left(\delta_{0}\right)$ is at most $n$. By [2] Theorem 3.4 there is a set $\delta_{1} \cong \delta_{0}$ such that the multiplicity of $E_{2}\left(\delta_{1}\right)$ is uniform and equal to that of $E_{2}\left(\delta_{0}\right)$. By applying the first part of the proof to the sub-space $E_{2}\left(\delta_{1}\right) X_{2}$ we will be able to find a Borel set $\delta_{2} \subseteq \delta_{1}$ such that $E_{1}\left(\delta_{2}\right) \neq 0$ and its multiplicity is not greater than that of $E_{2}\left(\delta_{0}\right)$. Since the multiplicity of $E_{1}\left(\delta_{2}\right)$ must be equal to $n$ the multiplicity of $E_{2}\left(\delta_{0}\right)$ will be exactly $n$.

Let us denote

$$
E_{2}\left(A_{0}\right)=\vee\left\{E_{2}(\sigma) \mid E_{2}(\sigma) \text { has uniform multiplicity } n\right\} .
$$

Obviously, $E_{2}\left(\Lambda_{0}\right)$ has uniform multiplicity $n$ and if $E_{2}\left(\Lambda_{0}\right) \neq I$, then, by repeating the arguments already used in the first part of the proof, one shall find a set $\sigma_{0}$ such that $E_{2}\left(\sigma_{0}\right)$ has multiplicity $n$ and $E_{2}\left(\sigma_{0}\right) \leqq I-E_{2}\left(\Lambda_{0}\right)$. Further, by [2] Theorem 3.4 there is a set $\sigma_{1} \subseteq \sigma_{0}$ such that $E_{2}\left(\sigma_{1}\right)$ has uniform multiplicity $n$. Hence $E_{2}\left(\Lambda_{0}\right)=I$ and the identity on $X_{2}$ would have uniform multiplicity $n$.

Assume that $E_{1}(\eta)$ has infinite uniform multiplicity for some Borel set $\eta$ (as we have already remarked, it must be equal to $\boldsymbol{\aleph}_{0}$ ). If $E_{2}(\eta)$ has not infinite uniform multiplicity then, by [2] Theorem 3.4, 
one can find a projection $0 \neq E_{2}\left(\eta_{1}\right) \leqq E_{2}(\eta)$ having finite uniform multiplicity. By the previous part of the proof $E_{1}\left(\eta_{1}\right)$ will also have finite multiplicity which contradicts the uniformity of the multiplicity of $E_{1}(\eta)$.

In order to prove the general assertion of the theorem, let us consider the decomposition of the identity (given by [2] Theorem 3.4) $I=\mathbf{V}_{1 \leqq n \leqq \aleph_{0}} E_{1}\left(\sigma_{n}\right)$ into disjoint projections such that if $E_{1}\left(\sigma_{n}\right) \neq 0, E_{1}\left(\sigma_{n}\right)$ has uniform multiplicity $n$. It follows that $I=\mathrm{V}_{1 \leqq n \leqq \boldsymbol{N}_{0}} E_{2}\left(\sigma_{n}\right)$ is the similar decomposition of the identity on $X_{2}$ (since this decomposition is unique). In conclusion the multiplicity of $E_{1}(\delta)$ is equal to that of $E_{2}(\delta)$ for every Borel set $\delta$ since

$$
E_{1}(\delta)=\bigvee_{1 \leqq n \leqq \aleph_{0}} E_{1}(\delta) E_{1}\left(\sigma_{n}\right) ; \quad E_{2}(\delta)=\bigvee_{1 \leqq n \leqq \boldsymbol{\aleph}_{0}} E_{2}(\delta) E_{2}\left(\sigma_{n}\right)
$$

and $E_{1}(\delta) E_{1}\left(\sigma_{n}\right)$ and $E_{2}(\delta) E_{2}\left(\sigma_{n}\right)$ have the same uniform multiplicity; $1 \leqq n \leqq \aleph_{0}$.

Let $A$ be a spectral operator whose resolution of the identity is $E(\cdot)$. By Foguel [6], the opeartor $A$ has a unique decomposition

$$
A=\int(\Re \mathfrak{e} \lambda) E(d \lambda)+i \int(\Im \mathfrak{m} \lambda) E(d \lambda)+N
$$

where $N$ is the generalized nilpotent part and $R=\Re e A=\int(\Re e \lambda) E(d \lambda)$ and $J=\Im \mathfrak{m} A=\int(\Im \mathfrak{m} \lambda) E(d \lambda)$ are called the real, respective, imaginary part of $A$ (this decomposition is similar to that of a normal operator into a sum of two commutative self-adjoint operators). Using these concepts we shall give the following necessary and sufficient condition for quasi-similarity.

THEoRem 6. The condition (ii) of Definition 1 can be replaced by (iii)

$$
\tau R_{1} \tau^{-1} y=R_{2} y ; \tau J_{1} \tau^{-1} y=J_{2} y ; \quad y \in D\left(\tau^{-1}\right)
$$

where $R_{i}$ and $J_{i}$ denote the real, respective, imaginary parts of $A_{i}$; $i=1,2$.

Proof. The implication (ii) $\Rightarrow$ (iii) was proved in Theorem 3 part (e). In order to prove the converse implication let us observe that from (iii) it follows that

$$
\tau \int p(\lambda, \bar{\lambda}) E_{1}(d \lambda) \tau^{-1} y=\int p(\lambda, \bar{\lambda}) E_{2}(d \lambda) y ; \quad y \in D\left(\tau^{-1}\right)
$$

for every polynomial $p(\lambda, \bar{\lambda})$. 
By the Stone-Weierstrass theorem, since $\tau$ is closed we get immediately

$$
\tau \int h(\lambda) E_{1}(d \lambda) \tau^{-1} y=\int h(\lambda) E_{2}(d \lambda) y ; \quad y \in D\left(\tau^{-1}\right)
$$

for every function $h$ continuous on a compact set $A$ containing the spectra of $A_{1}$ and $A_{2}$.

Let $\sigma \subseteq \Lambda$ be a closed set. By Urysohn's lemma (e.g. see [4] I-5-2) there exists, for each $m \geqq 1$, a continuous function $u_{m}$ with $0 \leqq u_{m}(\lambda) \leqq 1 ; u_{m}(\lambda)=1$ for $\lambda \in \sigma$ and $u_{m}(\lambda)=0$ when

$$
\min _{\mu \in \sigma}|\lambda-\mu| \geqq \frac{1}{m} \text {. }
$$

Obviously, $\left\{u_{m}(\lambda)\right\}$ converges to the characteristic function $\chi_{\sigma}(\lambda)$ of the set $\sigma$. Hence, by [4] IV-10-10, since $\tau$ is closed we have

$$
\tau E_{1}(\sigma) \tau^{-1} y=E_{2}(\sigma) y ; \quad y \in D\left(\tau^{-1}\right)
$$

for every closed set $\sigma$. If $\delta$ is a general Borel set, one can find a sequence of closed set $\left\{\sigma_{n}\right\}$ which increases to $\delta$. In view of the strong additivity of both resolutions of the identity we shall obtain

$$
\tau E_{1}(\delta) \tau^{-1} y=E_{2}(\delta) y ; \quad y \in D\left(\tau^{-1}\right)
$$

which implies (ii).

Corollary 7. Let $A_{1}$ and $A_{2}$ be two spectral operators with real spectrum. Then they are quasi-similar if and only if there exists a densely defined closed linear map $\tau: P_{1} \rightarrow P_{2}$ with densely defined inverse such that $\tau A_{1} \tau^{-1} y=A_{2} y ; y \in D\left(\tau^{-1}\right)$.

3. The Weyr characteristic. In this section we shall deal only with spectral operators whose resolutions of the identity contain no projections of infinite uniform multiplicity. This restriction is necessary in order to define the Weyr characteristic (after E. Weyr [11]). In fact, the Weyr characteristic was defined by Feldzamen in [5] for nilpotent operators commuting with Boolean algebras of projections of finite uniform multiplicity but his definition and the related results can be obtained without difficulties for nilpotent operators commuting with Boolean algebras of projections which contain no projections of uniform multiplicity.

We shall start by reproducing briefly (in our more general context) some definitions and results from [5] which concern the Weyr characteristic.

Let $\mathfrak{B}$ be a countably decomposable complete Boolean algebra of 
projections on a Banach space $X$ containing no projections of infinite uniform multiplicity. It is well known that $\mathfrak{B}$ can be considered as a spectral measure $E(\cdot)$ on its own support space $\Omega$. A Borel set $\delta$ of $\Omega$ will be called negligible if $E(\delta)=0$.

Let us consider a nilpotent operator $Q$ commuting with $\mathfrak{B}$.

A family of vectors $\left\{x_{\alpha} \mid \alpha \in A\right\}$ will be called a $k^{\text {th }}$ index system over $\delta$, for an integer $k$ and nonnegligible set $\delta$, if

(a) $Q^{k} x_{\alpha}=0 ; \quad \alpha \in A$

(b) $C\left(Q^{k-1} x_{\alpha}\right) \geqq E(\delta) ; \quad \alpha \in A$

(c) $\left\{Q^{i} x_{\alpha} \mid i=0,1, \cdots, k-1, \alpha \in A\right\}$ is independent.

By [2] Theorem 3.4 every nonnegligible set $\delta$ contains a nonnegligible subset $\sigma$ such that $E(\sigma)$ has finite uniform multiplicity. Then $\left\{E(\sigma) Q^{i} x_{\alpha} \mid i=0,1, \cdots, k-1, \alpha \in A\right\}$ is an independent family of vectors from $E(\sigma) X$ and hence, by [5] Theorem 6, it is finite.

Repeating the proof of [5] Theorem 16 we can show that for each integer $k$ and nonnegligible Borel set $\delta \subseteq \Omega$, two maximal $k^{t h}$ index systems over $\delta$ have the same cardinality.

The Weyr characteristic of $Q$ with respect to $\mathfrak{B}$, written $\mathscr{Y}(Q, \delta, k)$, will be defined as the cardinality of a maximal $k^{\text {th }}$ system over $\delta$ if $\delta$ is nonnegligible and zero whenever it is negligible.

Another result of Feldzamen which can be easily adapted in our case asserts that if $\delta$ is the disjoint union of a countable family $\left\{\delta_{i}\right\}$ of nonnegligible sets, then

$$
\mathscr{W}(Q, \delta, k)=\min _{i}\left\{\mathscr{W}\left(Q, \delta_{i}, k\right)\right\}
$$

for each $k$.

This theorem together with [2] Theorem 3.4 will be our basic tools. They will allow us to pass from the finite uniform multiplicity case to the general case by using Feldzamen's results from [5] without modifications.

THEOREM 8. The Weyr characteristic is a quasi-similarity invariant, i.e., if $A_{1}$ and $A_{2}$ are quasi-similar spectral operators, then

$$
\mathscr{W}\left(N_{1}, \delta, k\right)=\mathscr{W}\left(N_{2}, \delta, k\right)
$$

for each integer $k$ and Borel set on the complex plane.

Proof. In view of the previous remark and Theorem 5 it is enough to prove (3.3) for sets $\delta$ for which $E_{1}(\delta)$ and $E_{2}(\delta)$ have the same finite uniform multiplicity $n$. In order to simplify the notations 
we shall assume that both resolutions of the identity of $A_{1}$ and $A_{2}$ are Boolean algebras of projections of finite uniform multiplicity $n$.

For every nonnegligible set $\delta$ and integer $k$ we shall consider the family of all $k^{\text {th }}$ index systems over $\delta$ (with respect to $N_{1}$ and $E_{1}(\cdot)$ ) which contain only vectors from $D(\tau)$ where $\tau$ is the map defining the quasi-similarity between $A_{1}$ and $A_{2}$. By repeating the proof of [5] Theorem 16 one can show that two such maximal systems (composed from vectors of $D(\tau)$ ) have the same cardinality. Hence, we can define the Weyr characteristic with respect to $D(\tau)$ as the cardinality of a maximal $k^{\text {th }}$ index system containing only vectors from $D(\tau)$. It will be denoted by $\mathscr{W}_{0}\left(N_{1}, \delta, k\right)$. In the same way one can define (for $N_{2}$ and $E_{2}(\cdot)$ ) the Weyr characteristic with respect to $D\left(\tau^{-1}\right)$ which will be denoted by $\mathscr{\mathscr { K }}_{0}\left(N_{2}, \delta, k\right)$. Evidently,

$$
\mathscr{W}_{0}\left(N_{i}, \delta, k\right) \leqq \mathscr{W}\left(N_{i}, \delta, k\right) ; \quad i=1,2 .
$$

for every Borel set $\delta$ and integer $k$. Let us also remark that if $\left\{x_{1}, \cdots, x_{\alpha} \mid \alpha \in A, x_{\alpha} \in D(\tau)\right\}$ is a maximal $k^{\text {th }}$ index system over $\delta$ with respect to $D(\tau)$ then, by Theorem 3 part (e) $\left\{\tau x_{1}, \cdots, \tau x_{\alpha}\right\}$ is a $k^{\text {th }}$ index system over $\delta$ with respect to $D\left(\tau^{-1}\right)$ i.e. $\mathscr{W}_{0}\left(N_{1}, \delta, k\right) \leqq$ $\mathscr{W}_{0}\left(N_{2}, \delta, k\right)$ and by means of symmetry the reverse is also true. Consequently,

$$
\mathscr{W}_{0}\left(N_{1}, \delta, k\right)=\mathscr{W}_{0}\left(N_{2}, \delta, k\right)
$$

for every Borel set $\delta$ and integer $k$.

Let $\delta_{0}$ be a set of uniform characteristic for $N_{1}$ i.e. $\mathscr{W}\left(N_{1}, \delta_{0}, k\right)=$ $\mathscr{W}\left(N_{1}, \eta, k\right)$ for $k=0,1, \cdots, n$ and every nonnegligible set $\eta \subset \delta_{0}$. We shall prove that there exists a nonnegligible set $\sigma \subset \delta_{0}$ such that

$$
\sum_{k=1}^{n} \mathscr{\mathscr { V }}_{0}\left(N_{1}, \sigma, k\right)=n \text {. }
$$

First, let us observe that by [5] Corollary 20 and (3.4)

$$
\sum_{k=1}^{n} \mathscr{W}_{0}\left(N_{1}, \eta, k\right) \leqq n
$$

for every nonnegligible set $\eta \cong \delta_{0}$. Hence, we can define

$$
l=\max _{0 \neq \eta \subset \delta_{0}} \sum_{k=1}^{n} \mathscr{W}_{0}\left(N_{1}, \eta, k\right) .
$$

If $l=n$ the proof is finished; thus we can assume that $l<n$ and choose a nonnegligible set $\sigma \subseteq \delta_{0}$ such that

$$
\sum_{k=1}^{n} \mathscr{W}_{0}\left(N_{1}, \sigma, k\right)=l \text {. }
$$


Suppose that $m ; m \leqq n$ is the maximum integer for which

$$
\mathscr{\mathscr { V }}_{0}\left(N_{1}, \sigma, m\right) \neq 0,
$$

and let $\left\{z_{\alpha}^{m} \mid z_{\alpha}^{m} \in D(\tau), \alpha \in A_{m}\right\}$ be a maximal $m^{\text {th }}$ index system over $\sigma$ with respect to $D(\tau)$ for which $C\left(z_{\alpha}^{m}\right)=E_{1}(\sigma)$ for each $\alpha \in A_{m}$. Since $\left\{N_{1} z_{\alpha}^{m} \mid \alpha \in A_{m}\right\}$ is a $(m-1)^{\text {th }}$ index system over $\sigma$ with respect to $D(\tau)$ (see, e.g., Theorem 3 and [5] Theorem 17 (a)) there is a maximal $(m-1)^{\text {th }}$ index system over $\sigma$ with respect to $D(\tau)$ containing $\left\{N_{1} z_{\alpha}^{m} \mid \alpha \in A_{m}\right\}$, say $\left\{z_{\alpha}^{m-1} \mid z_{\alpha}^{m-1} \in D(\tau), \alpha \in A_{m-1}\right\}$ with $C\left(z_{\alpha}^{m-1}\right)=E_{1}(\sigma)$ for each $\alpha \in A_{m-1}$. Continuing so, we shall get a family of vectors $\left\{z_{\alpha}^{k} \mid z_{\alpha}^{k} \in D(\tau), \alpha \in A_{k}, k=1, \cdots, m\right\}$, whose cardinality is $l$. This family, which will be called a complete index system over $\sigma$ with respect to $D(\tau)$ is independent (the proof of [5] Theorem 19 for general complete index systems over $\sigma$ can be immediately adapted in our case). Let us remark that the set $E_{1}(\sigma) D(\tau)$ cannot be contained in

$$
\vee\left\{\mathfrak{M}\left(z_{\alpha}^{k}\right) \mid \alpha \in A_{k}, k=1, \cdots, m\right\}
$$

otherwise the multiplicity of $E_{1}(\sigma)$ will be equal to $l<n$. Thus, there is a vector $z \in D(\tau), C(z)=E_{1}\left(\sigma_{1}\right) \neq 0 ; \sigma_{1} \subseteq \sigma$ such that the family $\left\{z, z_{\alpha}^{k} \mid \alpha \in A_{k}, k=1, \cdots, m\right\}$ is independent. Let $j$ be such that $N_{1}^{j} z=0 ; N_{1}^{j-1} z \neq 0$ and suppose that the family

$$
\left\{N^{i} z, z_{\alpha}^{k} \mid \alpha \in A_{k}, k=1, \cdots, j, i=0,1, \cdots, j-1\right\}
$$

is independent. Then the vectors $\left\{z, z_{\alpha}^{j} \mid \alpha \in A_{j}\right\}$ form a $j^{\text {th }}$ index system over $\sigma_{1}$ with respect to $D(\tau)$ and therefore

$$
\mathscr{W}_{0}\left(N_{1}, \sigma_{1}, j\right)>\mathscr{W}_{0}\left(N_{1}, \sigma, j\right) .
$$

Since $\mathscr{W}_{0}\left(N_{1}, \sigma_{1}, k\right) \geqq \mathscr{W}_{0}\left(N_{1}, \sigma, k\right) ; k=1, \cdots, m$ we have

$$
\sum_{k=1}^{n} \mathscr{W}_{0}\left(N_{1}, \sigma_{1}, k\right)>l
$$

which contradicts the maximality of $l$. Hence the set

$$
\left\{N^{i} z, z_{\alpha}^{k} \mid \alpha \in A_{k}, k=1, \cdots, j, i=0,1, \cdots, j-1\right\}
$$

is not independent. From this point we can follow the arguments used in the proof of [5] Theorem 21 where, instead of obtaining a contradiction to the uniformity of the Weyr characteristic we shall contradict the maximality of $l$. Thus $l=n$ and (3.6) holds for the set $\sigma$ which satisfies (3.8).

Let us assume that there is a set $\hat{o}$ and an integer $k_{0}$ for which (3.3) does not hold. With no loss of generality we can suppose that

$$
\mathscr{W}\left(N_{1}, \delta, k_{0}\right)<\mathscr{W}\left(N_{2}, \delta, k_{0}\right) \text {. }
$$


By [5] Theorem 18 there is a partitioning of $\delta, \delta=\bigcup_{j=1}^{s} \delta_{j}$ as a disjoint finite union of sets of uniform characteristic $\mathscr{Y}\left(N_{1}, \cdot, \cdot\right)$. By [5] Theorem 17 (c)

$$
\mathscr{\mathscr { V }}\left(N_{i}, \delta, k_{0}\right)=\min _{1 \leqq j \leqq s} \mathscr{W}\left(N_{i}, \hat{o}_{j}, k_{0}\right) ; \quad i=1,2 .
$$

Thus, there is a set $\delta_{0} \subseteq \delta$ having uniform characteristic $\mathscr{W}\left(N_{1}, \cdot, \cdot\right)$, for which

$$
\mathscr{W}\left(N_{1}, \hat{o}_{0}, k_{0}\right)<\mathscr{W}\left(N_{2}, \hat{o}_{c}, k_{0}\right) \text {. }
$$

But, by the previous part of the proof (3.6) there exists a set $\sigma \subset \delta_{0}$ such that

$$
\sum_{k=1}^{n} \mathscr{W}_{0}\left(N_{1}, \sigma, k\right)=n
$$

and further, by (3.4) and [5] Theorem 21

$$
\mathscr{W}_{0}\left(N_{1}, \sigma, k\right)=\mathscr{\mathscr { V }}\left(N_{1}, \sigma, k\right) ; \quad k=1, \cdots, n .
$$

On the other hand, using (3.5), (3.4) and [5] Corollary 20 we have

$$
n=\sum_{k=1}^{n} \mathscr{W}_{0}\left(N_{2}, \sigma, k\right) \leqq \sum_{k=1}^{n} \mathscr{W}^{-}\left(N_{2}, \sigma, k\right) \leqq n
$$

i.e.

$$
\mathscr{W}_{5}\left(N_{2}, \sigma, k\right)=\mathscr{\mathscr { V }}\left(N_{2}, \sigma, k\right) ; \quad k=1, \cdots, n .
$$

In conclusion, by (3.5), (3.11) and (3.12) we shall get

$$
\mathscr{W}\left(N_{1}, \sigma, k_{0}\right)=\mathscr{W}\left(N_{2}, \sigma, k_{0}\right)
$$

and further, in view of the uniformity of $\delta_{0}$ and [5] Theorem 17 (b)

$$
\mathscr{W}^{-}\left(N_{1}, \hat{o}_{0}, k_{0}\right) \geqq \mathscr{W}^{-}\left(N_{2}, \delta_{0}, k_{0}\right)
$$

which contradicts $(3.10)$.

Lemma 9. Let $\mathfrak{B}$ be a countably decomposable complete Boolean algebra of projections having finite uniform multiplicity $n$ and $\left\{x_{1}, \cdots, x_{k}\right\}$ a set of $k ; 1 \leqq k \leqq n$ independent vectors such that $C\left(x_{i}\right)=I ; i=1, \cdots, k$. Then, there are $n-k$ vectors $x_{k+1}, \cdots, x_{n}$ such that

$$
\hat{w}=\bigvee_{i=1}^{n} \mathfrak{M}\left(x_{i}\right)
$$

Proof. Let us consider all the independent families of vectors $\left\{x_{1}, \cdots, x_{k+1}, y_{k+1}, \cdots, y_{p}\right\}$ for which $\bigwedge_{i=k+1}^{p} C\left(y_{i}\right) \neq 0$. By [5] Theorem 6 
we have $p \leqq n$; hence we can find such a system $\left\{x_{1}, \cdots, x_{k}, y_{k+1}, \cdots, y_{p}\right\}$ with $p$ maximal. Let us denote $E_{0}=\Lambda_{i=k+1}^{p} C\left(y_{i}\right)$ and

$$
\mathfrak{M}=\operatorname{clm}\left\{\mathfrak{M}\left(E_{0} x_{i}\right), \mathfrak{M}\left(E_{0} y_{2}\right) \mid 1 \leqq i \leqq k, k+1 \leqq j \leqq p\right\} .
$$

If there is $x_{0} \in X$ such that $E_{0} x_{0} \notin \mathfrak{M}$ then by [2] Lemma 4.7 one can find a maximal projection $E_{1} \in \mathfrak{B}$ such that $E_{1} E_{0} x_{0} \in \mathfrak{M} \mathfrak{l}$ and

$$
\mathfrak{M} \cap \mathfrak{M}\left(\left(I-E_{1}\right) E_{0} x_{0}\right)=\{0\} .
$$

Evidently, $y_{p+1}=\left(I-E_{1}\right) E_{0} x_{0} \neq 0$ and $0 \neq C\left(y_{p+1}\right) \leqq E_{0}$ i.e. in the family $\left\{E_{0} x_{1}, \cdots, E_{0} x_{k}, E_{0} y_{k+1}, \cdots, E_{0} y_{p}, E_{0} y_{p+1}\right\}$ the lower bound of the carrier projections is different from zero and by [5] Theorem 8 this family will be independent. This fact contradicts the maximality of $p$, thus $E_{0} X=\mathfrak{M}$ and further $p=n$ since $E_{0}$ has multiplicity $n$.

Let $\mathfrak{B}_{0}$ be the set of all the projections $0 \neq E \in \mathfrak{B}$ for which there are vectors $z_{k+1}, \cdots, z_{n}$ such that

$$
E X=\operatorname{clm}\left\{\mathfrak{M}\left(E x_{1}\right), \cdots, \mathfrak{M}\left(E x_{k}\right), \mathfrak{M}\left(E z_{k+1}\right), \cdots, \mathfrak{M}\left(E z_{n}\right)\right\} .
$$

By the previous part of the proof $\mathfrak{B}_{0}$ is not void. Let us put

$$
F=\vee\left\{E \mid E \in \mathfrak{B}_{0}\right\}
$$

and consider families of disjoint projections in $\mathfrak{B}$ each member of which is bounded by a projection $E \in \mathfrak{B}_{0}$, and order these families by inclusion. By Zorn's lemma there exists a maximal family which must be countable since $\mathfrak{B}$ is countably decomposable. Obviously, the union of this maximal family is $F$ i.e.

$$
F=\bigvee_{\nu=1}^{\infty} F_{2}
$$

and $F_{2} \in \mathfrak{B}_{0}$ since it is bounded by a projection $E_{2} \in \mathfrak{B}_{0}$. Thus there exist vectors $z_{k+1}^{(\nu)}, \cdots, z_{n}^{(\nu)} ; \nu=1,2, \cdots$ such that

$$
\begin{aligned}
F_{2} X=\operatorname{clm}\left\{\mathfrak{M C}\left(F_{\nu} x_{1}\right), \cdots, \mathfrak{M c}\left(F_{\nu} x_{k}\right), \mathbb{M}_{i}\left(F_{\nu} z_{k+1}^{(\nu)}\right), \cdots, \mathfrak{M}\left(F_{\nu} z_{n}^{(\nu)}\right)\right\} ; \\
\nu=1,2, \cdots .
\end{aligned}
$$

If we denote

$$
z_{3}=\sum_{\nu=1}^{\infty} \frac{F_{\nu} z_{j}^{(\nu)}}{2^{\nu}\left\|F_{\nu} z_{j}^{(\nu)}\right\|}, \quad j=k+1, \cdots, n
$$

then it is easy to see that

$$
F X=\operatorname{clm}\left\{\mathfrak{M}\left(F x_{1}\right), \cdots, \mathfrak{M}\left(F x_{k}\right), \mathfrak{M}\left(F z_{k+1}\right), \cdots, \mathfrak{M}\left(F z_{n}\right)\right\}
$$

i.e. $F \in \mathfrak{B}_{0}$.

If $F \neq I$, then by applying the first part of the proof on the 
subspace $(I-F) X$ we shall find a projection $0 \neq F_{0} \in \mathfrak{B}_{0} ; F_{0} \leqq I-F_{0}$ which constitutes a contradiction since $F_{0} \leqq F$.

Lemma 10. If $\mathscr{W}\left(N_{1}, \delta, k\right)=\mathscr{W}\left(N_{2}, \delta, k\right)$ for each integer $k$ and Borel set $\delta$ then the multiplicities of $E_{1}(\delta)$ and $E_{2}(\delta)$ are equal for every Borel set $\delta$.

Proof. First, let us consider a set $\eta$ such that both $E_{1}(\eta)$ and $E_{2}(\eta)$ have (finite) uniform multiplicity. By [5] Theorem $18 \eta$ may be decomposed as a union

$$
\eta=\bigcup_{j=1}^{s} \eta_{j}
$$

of a disjoint finite family of sets each of them having uniform characteristic (with respect to the Weyr characteristics $\mathscr{Y}\left(N_{1}, \cdot, \cdot\right)$ and $\left.\mathscr{Y}\left(N_{2}, \cdot, \cdot\right)\right)$. In order to simplify the notation we shall denote both multiplicity functions by $m(\cdot)$. Then, by [5] Theorem 21

$$
\begin{array}{rr}
m\left(E_{1}\left(\eta_{j}\right)\right)=\sum_{k=1}^{\infty} \mathscr{W}\left(N_{1 \mid E_{1}\left(\eta_{j}\right) X_{1}}, \eta_{i}, k\right)=\sum_{k=1}^{\infty} \mathscr{W}\left(N_{1}, \eta_{j}, k\right)=\sum_{k=1}^{\infty} \mathscr{W}\left(N_{2}, \eta_{j}, k\right) \\
=\sum_{k=1}^{\infty} \mathscr{\mathscr { V }}\left(N_{2 / E_{2}\left(\eta_{j}\right) X_{2}}, \eta_{j}, k\right)=m\left(E_{2}\left(\eta_{\jmath}\right)\right) ; & j=1, \cdots, s,
\end{array}
$$

and further, by [2] Lemma $3.3 m\left(E_{1}(\eta)\right)=m\left(E_{2}(\eta)\right)$.

For every set $\delta$, let

$$
\delta=\bigcup_{n=1}^{\infty} \hat{o}_{n}^{(1)} ; \quad \delta=\bigcup_{n=1}^{\infty} \delta_{n}^{(2)}
$$

be the decompositions of $\delta$ as a union of disjoint countable family of sets given by [2] Theorem 3.4 (for the Boolean algebras of projections $E_{1}(\cdot)$ and $E_{2}(\cdot)$, respectively). Then a set $\delta_{n}^{(1)} \cap \delta_{p}^{(2)}$ either is negligible or it has uniform multiplicity with respect to both multiplicity functions. Hence, by the first part of the proof $m\left(E_{1}\left(\delta_{n}^{(1)} \cap\left(\delta_{p}^{(2)}\right)\right)=\right.$ $m\left(E_{2}\left(\delta_{n}^{(1)} \cup \delta_{p}^{(2)}\right)\right)$ which implies $m\left(E_{1}(\delta)\right)=m\left(E_{2}(\delta)\right)$.

LEMma 11. Assume that $E_{1}(\cdot)$ and $E_{2}(\cdot)$ have the same finite uniform multiplicity $n$ and $\mathscr{W}\left(N_{1}, \hat{o}, k\right)=\mathscr{W}\left(N_{2}, \delta, k\right)$ for each integer $k$ and Borel set $\delta$. Then $A_{1}$ and $A_{2}$ are quasi-similar.

Proof. By Lemma $10 E_{1}(\cdot)$ and $E_{2}(\cdot)$ have the same support $\Lambda$. First, let us suppose that $\Lambda$ is a set of uniform characteristic with respect to $\mathscr{W}\left(N_{1}, \cdot, \cdot\right)$ and therefore to $\mathscr{W}\left(N_{2}, \cdot, \cdot\right)$ too. Let $m$ be the maximum integer for which the Weyr characteristics are not zero and $\left\{x_{\alpha}^{k} \mid x_{\alpha}^{k} \in X_{1} ; \alpha \in A_{k} ; k=1, \cdots, m\right\}$ and $\left\{y_{\alpha}^{k} \mid y_{\alpha}^{k} \in X_{2} ; \alpha \in A_{k} ; k=1, \cdots, m\right\}$ complete index systems over $\Lambda$ for $N_{1}$ respectively $N_{2}$ (see [5] after 
Theorem 18). In view of our hypotheses and [5] Theorem 21 both these systems have $n$ vectors. By [5] Theorem 19 and our Lemma 9

$$
\begin{aligned}
& X_{1}=\vee\left\{\mathfrak{M}\left(x_{\alpha}^{k}\right) ; \alpha \in A_{k} ; k=1, \cdots, m\right\} \\
& X_{2}=\vee\left\{\mathfrak{M}\left(y_{\alpha}^{k}\right) ; \alpha \in A_{k} ; k=1, \cdots, m\right\} .
\end{aligned}
$$

The principal property of these complete index systems consists in the fact that

$$
\begin{aligned}
& N_{1} x_{\alpha}^{k}=\left\{\begin{array}{l}
x_{\alpha}^{k-1} ; k>1 \\
0 ; k=1
\end{array} ; \quad N_{2} y_{\alpha}^{k}=\left\{\begin{array}{l}
y_{\alpha}^{k-1} ; k>1 \\
0 \quad ; k=1
\end{array} ;\right.\right. \\
& \alpha \in A_{k} ; k=1, \cdots m
\end{aligned}
$$

and thus, we can arrange them as follows:

$$
\begin{aligned}
& x_{1}^{m}, x_{1}^{m-1}=N_{1} x_{1}^{m}, x_{1}^{m-2}=N_{1}^{2} x_{1}^{m}, \cdots, x_{1}^{1}=N_{1}^{m-1} x_{1}^{m}, \\
& x_{2}^{m}, x_{2}^{m-1}=N_{1} x_{2}^{m}, x_{2}^{m-2}=N_{1}^{2} x_{2}^{m}, \cdots, x_{2}^{1}=N_{1}^{m-1} x_{2}^{m} \text {, }
\end{aligned}
$$

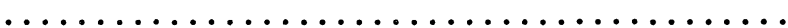

and in the same way for the second complete index system. Let $\left\{x_{1}, \cdots, x_{n}\right\}$ and $\left\{y_{1}, \cdots, y_{n}\right\}$ be the new order of those complete index systems given by the previous arrangements. Then

$$
X_{1}=\bigvee_{i=1}^{n} \mathfrak{M}\left(x_{i}\right) ; \quad X_{2}=\bigvee_{i=1}^{n} \mathfrak{M}\left(y_{i}\right) .
$$

For every set of $n$ Borel functions $\left\{f_{1}, \cdots, f_{n}\right\}$ for which $x_{i}$ belongs to the domain of the operator $\int f_{i}(\lambda) E_{1}(d \lambda)$ and $y_{i}$ to that of $\int f_{i}(\lambda) E_{2}(d \lambda) ; i=1, \cdots, n$ let us put

$$
\tau \sum_{i=1}^{n} \int f_{i}(\lambda) E_{1}(d \lambda) x_{i}=\sum_{i=1}^{n} \int f_{i}(\lambda) E_{2}(d \lambda) y_{i} .
$$

By [2] Theorem 5.2 the operator $\tau: X_{1} \rightarrow X_{2}$ and its inverse are closed densely defined. Further, it follows directly from the definition that $\tau E_{1}(\sigma) \tau^{-1} y=E_{2}(\sigma) y ; \sigma \in$ Borel sets and $y \in D\left(\tau^{-1}\right)$ which imply

$$
\left\|=E_{1}(\sigma) \tau^{-1} y\right\| \leqq M_{2}\|y\| ; \quad y \in D\left(\tau^{-1}\right)
$$

where $M_{2}$ is a bound of the resolution of the identity of $A_{2}$. In view of (3.14) and (3.15) we have

$$
\tau N_{1} \tau^{-1} y_{i}=N_{2} y_{i} ; \quad i=1, \cdots, n \text {. }
$$

and consequently $\tau N_{1} \tau^{-1} y=N_{2} y ; y \in D\left(\tau^{-1}\right)$. Since

$$
\tau S_{1} \tau^{-1} y=S_{2} y ; y \in D\left(\tau^{-1}\right)
$$

we can conclude that 


$$
\tau A_{1} \tau^{-1} y=A_{2} y ; \quad y \in D\left(\tau^{-1}\right)
$$

and the proof is complete in the case when $\Lambda$ has uniform characteristic. In the general case we can finish the proof by using [5] Theorem 18.

We shall proceed to prove the converse of Theorem 8 , i.e., we will show that the Weyr characteristic forms a complete set of invariants for quasi-similarity.

THEOREM 12. If the respective Weyr characteristics for $A_{1}$ and $A_{2}$ are equal, i.e.

$$
\mathscr{W}\left(N_{1}, \hat{o}, k\right)=\mathscr{Y}\left(N_{2}, \hat{o}, k\right)
$$

for each integer $k$ and Borel set $\delta$, then $A_{1}$ and $A_{2}$ are quasi-similar.

Proof. As before, let $\Lambda$ be the support of $E_{1}(\cdot)$ and $E_{2}(\cdot)$. By [2] Theorem 3.4 there is a unique decomposition of $A$ (for $E_{1}(\cdot)$ ),

$$
\Lambda=\bigcup_{n=1}^{\infty} \delta_{n}
$$

into disjoint Borel sets such that if $\delta_{n}$ is not negligible, $E_{1}\left(\hat{o}_{n}\right)$ has uniform multiplicity $n$. By Lemma $10, E_{2}\left(\delta_{n}\right)$ has also uniform multiplicity $n$. In view of Lemma 11, let

$$
\tau_{n}: E_{1}\left(\delta_{n}\right) X_{1} \longrightarrow E_{2}\left(\hat{o}_{n}\right) X_{2} ; \quad n=1,2, \cdots
$$

be the map defined in (3.16). Let us denote

$$
\begin{aligned}
& D(\tau)=\left\{x \mid x \in X_{1} ; E_{1}\left(\hat{o}_{n}\right) x \in D\left(\tau_{n}\right) ;\right. \\
& \left.n=1,2, \cdots \text { and } \sum_{n=1}^{\infty} \tau_{n} E_{1}\left(\hat{o}_{n}\right) x ; \text { converges }\right\}
\end{aligned}
$$

and

$$
\tau \varkappa=\sum_{n=1}^{\infty} \tau_{n} E_{1}\left(\delta_{n}\right) x
$$

Obviously, $\tau$ is a one-to-one densely defined map from $X_{1}$ into $X_{2}$ with densely defined inverse. Let $x_{p} \in D(\tau), p=1,2, \cdots$ and

$$
\lim _{p \rightarrow \infty} x_{p}=x ; \quad \lim _{p \rightarrow \infty} \tau x_{p}=y .
$$

Then,

$$
\lim _{p \rightarrow \infty} E_{1}\left(\delta_{n}\right) x_{p}=E_{1}\left(\delta_{n}\right) x ; \lim _{p \rightarrow \infty} E_{2}\left(\delta_{n}\right) \tau x_{p}=E_{2}\left(\delta_{n}\right) y ; \quad n=1,2, \cdots
$$

and further, $\lim _{p \rightarrow \infty} \tau_{n} E_{1}\left(\hat{o}_{n}\right) x_{p}=E_{2}\left(\delta_{n}\right) y$. Since $\tau_{n}$ is closed, 


$$
E_{1}\left(\delta_{n}\right) x \in D\left(\tau_{n}\right) \quad \text { and } \quad \tau_{n} E_{1}\left(\delta_{n}\right) x=E_{2}\left(\delta_{n}\right) y ; \quad n=1,2, \cdots
$$

which insures the convergence of $\sum_{n=1}^{\infty} \tau_{n} E_{1}\left(\delta_{n}\right) x=y$. Using the definition of $\tau$ we will obtain $\tau x=y$ i.e. $\tau$ is a closed map. Finally, the fact that $\tau$ defines a quasi-similarity between $A_{1}$ and $A_{2}$ follows immediately from Lemma 11.

COROLLARY 13. Quasi-similarity is an equivalence relation for spectral operators whose resolutions of the identity contain no projections of infinite uniform multiplicity.

Using the previous results and [5] Theorem 30 we have,

COROLlaRY 14. For spectral operators of finite multiplicity on Hilbert spaces, quasi-similarity coincides with Feldzamen's semisimilarity (see [5]).

From this corollary and [5] Theorem 27 (or [7] Theorem 3.1) we shall get

COROLlaRY 15. For normal operators of finite multiplicity on Hilbert spaces, quasi-similarity coincides with unitary equivalence.

CoRollary 16. Let $S_{1}$ and $S_{2}$ be two scalar operators whose resolutions of the identity $E_{1}(\cdot)$ and $E_{2}(\cdot)$ respectively, contain no projections of infinite uniform multiplicity. Then $S_{1}$ and $S_{2}$ are quasi-similar if and only if the multiplicities of $E_{1}(\delta)$ and $E_{2}(\delta)$ coincide for each Borel set $\delta$.

Proof. It is enough to observe that for scalar operators $\mathscr{W}(0, \delta, 1)$ is equal with the maximal number of independent vectors whose carrier is $E(\delta)$ while $\mathscr{W}(0, \delta, k)=0$ for $k>1$. Then, the proof can be finished by Theorems 8 and 12, Lemma 9, [5] Theorems 18 and 21, and [2] Theorem 3.4.

CoRollary 17. Let $S_{1}$ and $S_{2}$ be two self-adjoint operators on Hilbert spaces whose resolutions of the identity contain no projections of infinite uniform multiplicity. Then $S_{1}$ and $S_{2}$ are unitarily equivalent if and only if there exists a densely defined closed linear map $\tau: X_{1} \rightarrow X_{2}$ with densely defined inverse such that $\tau S_{1} \tau^{-1} y=$ $S_{2} y . y \in D\left(\tau^{-1}\right)$.

Proof. It follows from Corollaries 7 and 15. 
Finally, Bade's basic result [2] Theorem 9.2 can be reproduced as follows.

THEOREm 18. Let $X$ be a separable Banach space and $S$ be a scalar type operator in $X$. Suppose that the resolution of the identity $E(\cdot)$ of $S$ contains no projections of infinite uniform multiplicity. Then $S$ is quasi-similar to the "multiplication" operator on a suitable direct sum of $L_{1}$-spaces.

Following [5] we can introduce another characteristic, the Segre Characteristic, written $\mathscr{S}(\delta, k)$. It is defined to be the difference $\mathscr{W}(\delta, k)-\mathscr{W}(\delta, k+1)$ for each integer $k$ and Borel set $\delta$. One can easily see that $\mathscr{S}(\delta, k)$ completely determine the values of $\mathscr{\mathscr { T }}(\hat{o}, k)$. Hence the Segre characteristic (after C. Segre [9]) forms a second complete set of invariants of quasi-similarity.

Added in proof. An open question concerning the definition of quasi-similarity is whether condition (ii) in Definition 1 is redundant. We are indebted to Charles A. McCarthy for the following example showing that this condition is not superfluos.

Consider the $l_{2}$-space of all doubly-infinite sequences $\left\{a_{n}\right\}_{n}^{+\infty}{ }_{-\infty}$ with $\sum_{n=-\infty}^{+\infty}\left|a_{n}\right|^{2}<+\infty$ and define

$$
\tau\left\{a_{n}\right\}=\left\{a_{n} 2^{n}\right\} ; N\left\{a_{n}\right\}=\left\{a_{n+1}\right\} .
$$

Obviously, $\tau$ is a densely defined closed linear map with densely defined inverse and $N$ is a normal bounded operator satisfying $\tau N \tau^{-1}=$ $2 N$. But $N$ and $2 N$ are not quasi-similar because they do not have the same spectrum (see Corollary 4).

\section{REFERENCES}

1. W. G. Bade, On Boolean algebras of projections and algebras of operators, Trans. Amer. Math. Soc. 80 (1955), 345-359.

2. - A multiplicity theory for Boolean algebras of projections on Banach spaces, Trans. Amer. Math. Soc. 92 (1959), 508-530.

3. N. Dunford, Spectral operators, Pacific J. Math. 4 (1954), 321-354.

4. N. Dunford and J. Schwartz, Linear Operators, New-York, Interscience Publishers; vol. I, 1958; vol. II, 1963; vol. III, to appear.

5. A. N. Feldzamen, Semi-similarity invariants for spectral operators on Hilbert space, Trans. Amer. Math. Soc. 100 (1961). 27i-323.

6. S. R. Foguel, The relations between a spectral operator and its scalar part. Pacific J. Math. 8 (1958), 51-65.

7. - On a paper of Feldzamen, Israel J. Math. 1 (1963), 133-138.

8. P. R. Halmos, Introduction to Hilbert Space and the Theory of Spectral Multiplicity, Chelsea, New-York, 1951.

9. C. Segre, Sulla teoria e sulla classificazione delle omografie in uno spazio lineare ad 
un numero qualunque di dimensioni, Atti Accad. naz. Lincei, Mem. (III) 19 (1884), 127-148.

10. L. Tzafriri, Operators commuting with Boolean algebras of projections of finite multiplicity, Pacific J. Math. 20 (1967), 571-587.

11. E. Weyr, Repartition des matrices en especes et formation de toutes les especes, C. R. Acad. Sci. Paris 100 (1885), 966-969.

Received September 1, 1966.

The Hebrew University of Jerusalem 



\section{PACIFIC JOURNAL OF MATHEMATICS}

\section{EDITORS}

H. ROYDEN

Stanford University

Stanford, California

\section{J. P. Jans}

University of Washington

Seattle, Washington 98105

\section{J. DUGUNDJI}

Department of Mathematics University of Southern California Los Angeles, California 90007

\section{RICHARD ARENS}

University of California

Los Angeles, California 90024

\section{ASSOCIATE EDITORS}

E. F. BECKENBACH

B. H. NEUManN

F. WOLF

K. YosIDA

\section{SUPPORTING INSTITUTIONS}

UNIVERSITY OF BRITISH COLUMBIA CALIFORNIA INSTITUTE OF TECHNOLOGY UNIVERSITY OF CALIFORNIA MONTANA STATE UNIVERSITY UNIVERSITY OF NEVADA NEW MEXICO STATE UNIVERSITY OREGON STATE UNIVERSITY UNIVERSITY OF OREGON OSAKA UNIVERSITY UNIVERSITY OF SOUTHERN CALIFORNIA
STANFORD UNIVERSITY

UNIVERSITY OF TOKYO UNIVERSITY OF UTAH WASHINGTON STATE UNIVERSITY UNIVERSITY OF WASHINGTON AMERICAN MATHEMATICAL SOCIETY CHEVRON RESEARCH CORPORATION TRW SYSTEMS NAVAL WEAPONS CENTER 


\section{Pacific Journal of Mathematics}

\section{Vol. 25, No. $1 \quad$ September, 1968}

Glen Eugene Bredon, Cosheaves and homology................... 1

Robin Ward Chaney, A chain rule for the transformation of integrals in

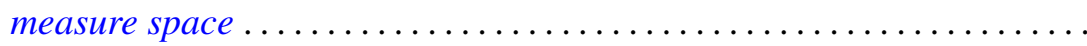

Colin W. Clark, On relatively bounded perturbations of ordinary differential operators................................... 59

John Edwin Diem, A radical for lattice-ordered rings.............. 71

Zeev Ditzian, On a class of convolution transforms ................ 83

Dennis Garoutte and Paul Adrian Nickel, A note on extremal properties characterizing weakly $\lambda$-valent principal functions............. 109

Shwu-Yeng Tzeng Lin, Fixed point properties and inverse limit spaces . . . 117

John S. Lowndes, Some dual series equations involving Laguerre polynomials ................................. 123

Kirti K. Oberai, Sum and product of commuting spectral operators ....... 129

J. N. Pandey and Armen H. Zemanian, Complex inversion for the generalized convolution transformation..................... 147

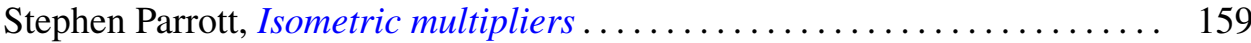

Manoranjan Prasad, Note on an extreme form .................. 167

Maciej Skwarczyński, A representation of a bounded function as infinite product in a domain with Bergman-Shilov boundary surface ......... 177

John C. Taylor, The Šilov boundary for a lattice-ordered semigroup ...... 185 Donald Reginald Traylor and James Newton Younglove, On normality and pointwise paracompactness ............................. 193

L. Tzafriri, Quasi-similarity for spectral operators on Banach spaces ... 